\title{
Brain Function Differences in Language Processing in Children and Adults with Autism
}

\author{
Diane L. Williams, Vladimir L. Cherkassky, Robert A. Mason, Timothy A. Keller, Nancy J. Minshew, \\ and Marcel Adam Just
}

\begin{abstract}
Comparison of brain function between children and adults with autism provides an understanding of the effects of the disorder and associated maturational differences on language processing. Functional imaging (functional magnetic resonance imaging) was used to examine brain activation and cortical synchronization during the processing of literal and ironic texts in 15 children with autism, 14 children with typical development, 13 adults with autism, and 12 adult controls. Both the children and adults with autism had lower functional connectivity (synchronization of brain activity among activated areas) than their age and ability comparison group in the left hemisphere language network during irony processing, and neither autism group had an increase in functional connectivity in response to increased task demands. Activation differences for the literal and irony conditions occurred in key language-processing regions (left middle temporal, left pars triangularis, left pars opercularis, left medial frontal, and right middle temporal). The children and adults with autism differed from each other in the use of some brain regions during the irony task, with the adults with autism having activation levels similar to those of the control groups. Overall, the children and adults with autism differed from the adult and child controls in (a) the degree of network coordination, (b) the distribution of the workload among member nodes, and (3) the dynamic recruitment of regions in response to text content. Moreover, the differences between the two autism age groups may be indicative of positive changes in the neural function related to language processing associated with maturation and/or educational experience. Autism Res 2013, ••: ••-••. (c) 2013 International Society for Autism Research, Wiley Periodicals, Inc.
\end{abstract}

Keywords: fMRI; language processing; development; functional connectivity

Brain imaging research in adults with autism, using functional magnetic resonance imaging (fMRI), has established that during language comprehension (one of the main types of cognition affected in autism) both the relative use and coordination of key brain regions differ systematically from that of individuals with typical development (TD). Adults with autism tend to overrely on posterior language regions; they activate these regions to a larger extent than typical controls, and these regions are less functionally connected to (synchronized with) the frontal regions, as follows. In the classic left hemisphere network, adults with autism demonstrate a posterior emphasis in their activation pattern with relatively greater temporal activation [Wernicke's area or left superior temporal gyrus (LSTG)] than frontal activation (Broca's area or left inferior frontal gyrus) in both sentence comprehension [Just, Cherkassky, Keller, \& Minshew, 2004] and in semantic decision making [Harris et al., 2006]. Furthermore, reduced frontal-posterior functional connectivity as compared with typical controls has been found for adults with autism during a number of language tasks [Just et al., 2004; Kana, Keller, Cherkassky, Minshew, \& Just, 2006; Mason, Williams, Kana, Minshew, \& Just, 2008]. Adults with autism also exhibit a lack of selective activation of relevant right hemispheric regions in response to increased sentence difficulty or the presence of intentionality (theory-of-mind) information during discourse comprehension [Mason et al., 2008]. However, much like adults with TD, adults with autism demonstrate spillover processing from the languagedominant left hemisphere to right hemisphere language homologs (i.e. right inferior frontal gyrus) in languageprocessing tasks that are relatively more difficult for them [Tesink et al., 2009]. In combination, these results indicate that, although similar cortical regions are used in language comprehension in autism as in neurotypical

From the Department of Speech Language Pathology, Duquesne University, Pittsburgh, Pennsylvania (D.L.W.); Center for Cognitive Brain Imaging, Department of Psychology, Carnegie Mellon University, Pittsburgh, Pennsylvania (D.L.W., V.L.C., R.A.M., T.A.K., M.A.J.); Departments of Psychiatry and Neurology, University of Pittsburgh School of Medicine, Pittsburgh, Pennsylvania (N.J.M.)

Received May 31, 2011; accepted for publication February 15, 2013

Address for correspondence and reprints: Diane L. Williams, Center for Cognitive Brain Imaging, Department of Psychology, Carnegie Mellon University, 5000 Forbes Avenue, Pittsburgh, PA 15213-3890. E-mail: diane72@andrew.cmu.edu

Grant Sponsor: National Institute of Child Health and Human Development.

Grant Numbers: Autism Center of Excellence HD055748; Collaborative Programs of Excellence in Autism U19HD35469

Grant Sponsor: National Institute of Deafness and Other Communication

Grant Number: K23 DC00669 [to DLW]

Published online in Wiley Online Library (wileyonlinelibrary.com)

DOI: $10.1002 /$ aur.1291

(c) 2013 International Society for Autism Research, Wiley Periodicals, Inc. 
participants, the brain affected by autism differs in three interesting respects: (a) the degree of network coordination; (b) the distribution of the workload among member nodes; and (c) the dynamic recruitment of regions in response to text content.

Although small in number, fMRI studies with children and adolescents have reported differences in the activation of key language regions and possible differences in the communication between brain regions in autism. Knaus, Silver, Lindgren, Hadjikhani, and Tager-Flusberg [2008] used a semantic integration and word-generation task with adolescents with autism spectrum disorder (ASD), ages 11-19, and age-matched controls. The ASD group had more activation in Broca's area; the pattern of activation was less lateralized than that of the TD group; and the ASD group had a pattern of more diffuse activation as compared with the TD group. In addition, the percent signal change in frontal and temporal areas was correlated in the control group but not in the ASD group, which was interpreted as indicating less communication between these critical language areas in the ASD group [Knaus et al., 2008]. In a study of irony processing, children and adolescents with ASD, 7-16 years of age, had significantly greater activation in the right inferior frontal gyral region, in the left superior and middle temporal regions, and the left postcentral gyrus as compared with an age- and intelligence quotient (IQ)-matched control group of TD children [Wang, Lee, Sigman, \& Dapretto, 2006]. Wang and colleagues interpreted the increased activation as the effortful use of normative neural circuitry associated with the processing involved in understanding the mental states. These studies suggest that children and adolescents with autism demonstrate difficulties in the neurofunctional basis of language processing, similar to those exhibited by the adults. However, the effect in particular brain regions appears to differ somewhat from what has been reported for adults groups. It is unknown whether these differences reflect developmental differences or differences related to the tasks that were used for these studies.

Language acquisition is a developmental process with significant changes occurring through adolescence and with continued growth through adulthood, reflective of cognitive and biological maturation. Not surprisingly, differences in neurofunctional measures between children and adults with TD have been reported in a number of studies of language processing [Booth et al., 2004; Sachs \& Gaillard, 2003]. Therefore, language processing may be differentially affected in children with autism as compared with adults, particularly because it is a neurodevelopmental disorder. To date, fMRI studies of language processing in autism have examined neurofunctional differences separately within adult [e.g. Harris et al., 2006; Just et al., 2004; Mason et al., 2008], or within child or adolescent groups [e.g. Colich et al., 2012;
Knaus et al., 2008; Wang et al., 2006] using different language tasks. Despite some initial evidence of overall similarities in the results for both adults and children with autism, the specific results differ from what has been reported for the adults with respect to the regions involved and levels of activation for these regions. To begin to address these central developmental issues, neurofunction needs to be examined in both children and adults with autism as they perform the same language task.

Examination of the differences across age groups, using the same language task, may help us understand not only what differs in language processing in autism, but also how these differences are affected by brain maturation and experience. The children and adults would be expected to differ in some predictable ways. In general, adults are more proficient in language than children, related both to maturation of brain function and experience. Therefore, although the adults would be expected to demonstrate some differences between easier and more challenging language-processing tasks, they would demonstrate indices of neurofunctional efficiency that are not seen in younger individuals. These indices would include predictable increases in activation and increases in functional connectivity (synchronization of key brain regions) in response to task difficulty. What is unknown is whether similar positive changes or differential use of neural resources are seen with maturation in individuals with autism. Using the same language task with children and adults with and without autism allows comparisons according to diagnostic group status as well as examination of developmental differences. Therefore, in our study, we examined brain function during text comprehension in two developmental age groups representing different stages of the developmental disorder of autism.

Text that contains irony is especially interesting in autism because, in addition to demanding a higher level of linguistic processing relative to literal processing, it requires mentalizing or the consideration of the speaker's intent [Sperber \& Wilson, 1981, 1995], a task that is known to be challenging for individuals with autism [Baron-Cohen, Leslie, \& Frith, 1985; Kana, Keller, Cherkassky, Minshew, \& Just, 2009]. Our study thus systematically varied the processing demands of texts, which either did or did not contain an ironic statement.

Both the children and adults with autism were predicted to differ from the neurotypical participants in the three respects seen in previous studies, that is, in the dynamic recruitment of regions in response to text content, in the distribution of workload among member nodes, and in the degree of network coordination (functional connectivity). Because complex language comprehension is a signature deficit in autism, and because autism is a developmental disorder, we examined how these three characteristics of language processing in 
autism are manifested during the comprehension of passages containing irony, and how these characteristics change between late childhood and adulthood. The goal was to learn about developmental differences in brain organization for language in autism.

\section{Method}

Participants

High-functioning individuals with autism and age- and IQ-matched controls in two age groups participated in the study. The participants included 15 children with autism [mean age 13.0 years, standard deviation $(\mathrm{SD})=1.7 \mathrm{]}, 14$ control children with TD (mean age 12.5 years, $\mathrm{SD}=1.5$ ), 13 adults with autism (mean age 24.9 years, $\mathrm{SD}=8.4$ ), and 12 typically developing adult control participants (mean age 21.0 years, $\mathrm{SD}=3.7$ ). Demographic information is given in Table 1 . All participants were native English speakers. (A number of additional participants were tested but excluded from analysis because of excessive head motion, as detailed later.) The diagnosis of autism was established using the Autism Diagnostic Interview-Revised [Lord, Rutter, \& LeCouteur, 1994], the Autism Diagnostic Observation Schedule [Lord et al., 2000], and confirmed by expert clinical opinion. All participants were required to be in good medical health. Four of the adult autism participants took medications on the day of the scan (two of these were taking selective serotonin reuptake inhibitors, one of these was taking allergy medication, and one was taking medication for the treatment of hyperaldosteronism). Potential participants with autism were excluded if they had an

Table 1. Age, IQ, handedness, and gender of the participants

\begin{tabular}{|c|c|c|c|c|}
\hline & Autism & Control & & \\
\hline a) Child groups & Mean (SD) & Mean (SD) & $t(27)$ & $P$ \\
\hline Age (years) & $13.0(1.7)$ & $12.5(1.5)$ & 0.907 & 0.373 \\
\hline VIQ & $102.9(10.2)$ & $107.6(10.7)$ & 1.21 & 0.236 \\
\hline PIQ & $104.9(20.3)$ & $109.6(9.4)$ & 0.803 & 0.429 \\
\hline FSIQ & $104.3(14.8)$ & $110.0(10.6)$ & 1.18 & 0.248 \\
\hline Handedness & 15 right: 0 left & $14: 0$ & & \\
\hline \multirow[t]{2}{*}{ Gender } & 13 male: 2 female & $12: 2$ & & \\
\hline & Autism & Control & & \\
\hline b) Adults groups & Mean (SD) & Mean (SD) & $t(23)$ & $P$ \\
\hline Age (years) & $24.9(8.4)$ & $21.0(3.7)$ & 1.49 & 0.149 \\
\hline VIQ & $107.1(12.8)$ & $111.3(7.1)$ & 0.99 & 0.330 \\
\hline PIQ & $110.2(7.8)$ & $115.3(6.8)$ & 1.70 & 0.102 \\
\hline FSIQ & $109.9(9.0)$ & $114.6(7.0)$ & 1.43 & 0.165 \\
\hline Handedness & 11 right: 2 left & 10 right: 2 left & & \\
\hline Gender & 11 male: 2 female & 11 male: 1 female & & \\
\hline
\end{tabular}

IQ, intelligence quotient; FSIQ, full-scale IQ; PIQ, performance IQ; VIQ, verbal IQ;SD, standard deviation. identifiable cause for their autism such as fragile $\mathrm{X}$ syndrome, tuberous sclerosis, or fetal cytomegalovirus infection, or were found to have evidence of prematurity, birth asphyxia, head injury, or a seizure disorder. Exclusions were based on neurologic history and examination, physical examination, and chromosomal analysis or metabolic testing, if indicated.

The control participants were community volunteers and were group-matched to the participants with autism on age, gender, race, and all three IQ scores, verbal, performance, and full-scale (FSIQ), as determined by the administration of the Wechsler Abbreviated Scales of Intelligence [Wechsler, 1999]. Potential control participants were screened by questionnaire, telephone, face-toface interview, and observation during initial testing, and were excluded if they had a current or past history of prematurity, psychiatric and neurologic disorders, birth injury, developmental delay, school problems, acquired brain injury, learning disabilities, or medical disorders with implications for the central nervous system. Exclusionary criteria also included a history in first-degree relatives of autism, developmental cognitive disorder, learning disability, affective disorder, anxiety disorder, schizophrenia, obsessive compulsive disorder, or other neurologic or psychiatric disorder thought to have a genetic component. One of the adult control participants took medication for the treatment of rheumatoid arthritis and a peptic ulcer on the day of the scan.

Handedness was determined with the Lateral Dominance Examination from the Halstead-Reitan Neuropsychological Test Battery [Reitan, 1985]. Two adult participants with autism and two adult control participants were left handed. The brain activation data from these left handers were clearly similar to their respective groups, and therefore, the data are not separated by handedness. All participants were Caucasian except for one adult participant with autism who was African-American. Ten of the adult participants with autism and nine of the adult control participants were included in the participant group for a previously reported fMRI study on language and imagery [Kana et al., 2006], and all of the adult participants in both groups were included in a previously reported fMRI study on inhibition [Kana, Keller, Minshew, \& Just, 2007]. Written informed consent was obtained from participants and/or their guardians, and written assent was obtained from all minor participants using procedures approved by the University of Pittsburgh Medical Center and Carnegie Mellon University Institutional Review Boards.

\section{Experimental Paradigm}

This study was an event-related fMRI study that assessed the brain activation and behavioral performance of the four groups when reading brief stories presented on a 
computer screen. There were two experimental conditions: a literal text condition and an irony text condition. Each condition consisted of nine stories, each three sentences in length. The first two sentences, which provided the context, were presented for $8,000 \mathrm{msec}$, followed by a fixation of 2,000 msec. The final sentence was a literal or ironic statement made by one of the characters in the story (hereafter referred to as the critical utterance), presented for $4,000 \mathrm{msec}$, followed by a fixation of $6,000 \mathrm{msec}$. Then the participants were given 7,000 msec (question displayed for 4,000 msec followed by an additional 3,000 msec fixation) to answer a yes/no question about this statement, which they responded to by pressing one-button mice in their right and left hands, respectively. There was a 3,000-msec rest between each full story. The stories of the two types were presented in a random order. In addition, a 24-sec fixation condition, of which there were four instances, was presented every seventh story, to provide a baseline measure of brain activation with which to compare each experimental condition. In this fixation condition, participants fixated on a centered asterisk without performing any task.

The stimuli were taken from materials that had been developed for another fMRI study of irony comprehension [Eviatar \& Just, 2006]. All of the ironic utterances were nonmetaphoric, with the character always saying the opposite of what they actually meant. [Norming data is reported in Eviatar \& Just, 2006.] All passages and critical utterances were matched for character and word length across passage types. Additionally, the passage types were matched on the number of positive and negative responses to the probes. In the following stimuli examples, critical utterances are shown in bold.

Literal: Johnny went on a hike with his brother. Suddenly he saw a huge snake next to his foot. He

said, "I am so scared." Was Johnny afraid?

Irony: Tommy was raking leaves into large mounds. His brother ran through the piles. Tommy said,

"You are a big help." Does Tommy think his

brother helped him?

Each participant practiced the task before going into the scanner. The practice for the adult participants consisted of six stories, two of each text condition, that were similar to but not identical to the ones presented in the scanner. The practice for the child participants consisted of four stories, two of each text condition. Both adult and children participants completed the practice file at least once. In some cases, additional practice was provided to ensure that the participant understood the task before moving into the scanner. Prior to testing in the scanner, each participant had an additional practice session in an MRI simulator, a full-scale replica of the Siemens Allegra 3T scanner used for this study, to assure their comfort in the MRI environment.

\section{Functional Imaging}

FMRI parameters. Experiments were run on a 3.0Tesla Siemens Allegra scanner (Siemens, Erlangen, Germany) using a circularly polarized transmit/receive head coil at the Brain Imaging Research Center of Carnegie Mellon University and the University of Pittsburgh. The stimuli were rear-projected onto a translucent plastic screen attached to the roof of the bore of the scanner. Participants viewed the screen through a mirror mounted on the head coil. For the functional imaging, an echoplanar pulse sequence was used with $\mathrm{TR}=1,000 \mathrm{msec}$, $\mathrm{TE}=30 \mathrm{~ms}$, and a flip angle of $60^{\circ}$. Sixteen oblique-axial slices were acquired in an interleaved sequence, with 5 -mm slice thickness, $1-\mathrm{mm}$ slice gap, and a $200 \times 200 \mathrm{~cm}$ FOV, and a $64 \times 64$ matrix, resulting in an in-plane resolution of $3.125 \times 3.125 \mathrm{~mm}$.

fMRI preprocessing. Preprocessing of the imaging data was carried out using SPM99 (Welcome Department of Cognitive Neurology, London, UK, http://www.fil. ion.ucl.ac.uk/spm). The first five volumes were discarded to allow the signal to reach steady state, and the images were corrected for slice acquisition timing using sinc interpolation to the first slice acquired in each volume. Head motion was estimated using a least-squares method and a six-parameter rigid-body model with the first volume as a reference, and the data were then resliced using sinc interpolation. Motion estimates were evaluated for each participant, and datasets with more than $3 \mathrm{~mm}$ motion in any direction were excluded from further analyses. This resulted in the exclusion of data for 24 children with autism, 7 control children, 8 adults with autism, and 2 control adults. The excluded children with autism were reliably younger in age (mean age $=11.82$, $\mathrm{SD}=1.40$ ) than the included children with autism [t(37) $=2.46, P=0.019]$ but were not reliably different in cognitive ability $[\mathrm{t}(37)=0.36, P=0.72$ for FSIQ]. The excluded adults with autism were reliably different from the included adults with autism in that they were younger $\quad[$ mean age $=17.5, \quad \mathrm{SD}=2.43) ; \mathrm{t}(19)=2.42$, $P=0.026]$ and on average lower functioning cognitively $[$ mean FSIQ $=89.75, \mathrm{SD}=12.89 ; \mathrm{t}(19)=4.23, P<0.0001]$.

The maximum motion estimates for the remaining included participants were submitted to a 2 (diagnosis) by 2 (age group) analysis of variance, which revealed no main effect of diagnosis $[\mathrm{F}(1,50)=0.53, P=0.47]$ no main effect of age group $[\mathrm{F}(1,50)=1.43, P=0.25]$ and no interaction $[\mathrm{F}(1,50)=0.34, P=0.56]$. Furthermore, there was no reliable difference between autism and control groups in the mean, range, or variance of the two alternative motion metrics proposed by Power and colleagues [2012]. The data were then normalized to the Montreal Neurological Institute (MNI) template, resampled to $2 \times 2 \times 2$ mm voxels, and smoothed with an 8-mm Gaussian kernel to decrease spatial noise. 
Distribution of activation. Activation was measured using blood oxygen level-dependent (BOLD) contrast. Statistical analysis was performed with SPM2 on individual and group data using the general linear model and Gaussian random field theory [Friston et al., 1995]. Highpass temporal filtering was applied in the model with a cutoff of $128 \mathrm{sec}$ to remove low-frequency drift in the time-series, and an AR(1) correction was applied to account for temporal correlations. The context and critical utterance sentences (for each text condition) were modeled with separate regressors created by convolving a boxcar function with the standard hemodynamic response function as specified in SPM. Statistical maps were superimposed on normalized T1-weighted images. An uncorrected height threshold of $P=0.001$ and an extent threshold of six $8-\mathrm{mm}^{3}$ voxels were used for within- and between-groups analyses. The SPM analysis was performed for the full model, including regressors for context and questions. Here we report the results for the critical utterances only (the third sentences of each story) because this is when the processing related to literal or ironic understanding is most likely to occur. Even though there is perhaps no way to ascertain that the ironic content (particularly for the autism group) is processed on the critical utterance rather than at some delayed rate, the use of a covariate analysis was used to correct for the latter possibility.

Functional region of interest definition. Functional regions of interest (ROIs) were defined to encompass the main clusters of activation in the group activation map for each group in both of the critical utterance sentence contrasts versus fixation (literal and irony). A spherical ROI with a radius of $8 \mathrm{~mm}$ was defined corresponding to each cluster, such that it best captured the activation of individual participants in all four groups for each of the two contrasts. The ROIs used in the analysis were the union of the eight spheres defined for the four groups in the two conditions. Labels were assigned to the functional ROIs with reference to the parcellation of the MNI single-subject T1-weighted dataset carried out by Tzourio-Mazoyer et al. [2002]. Eight functional ROIs defined in this manner were the left medial frontal gyrus (LMedFG), the left inferior frontal gyrus-left pars opercularis (LOPER) and left pars triangularis (LTRIA), the LSTG, the right prefrontal cortex (RPFC), the right temporal parietal junction (RTPJ), plus the bilateral middle temporal gyri (LMT and RMT), all of which have been related to language processing. An additional four ROIs, the left precentral gyrus, the left supplemental motor area, plus the bilateral occipital poles, were identified but were not included in the analyses because these are thought to be related to motor and sensory processing, not a focus of this study. Participants who did not have activation in a given functional ROI were excluded from further analysis involving that ROI as described in the description of the functional connectivity analyses later.

\section{Differences in beta weights (contrasts against}

fixation). To further characterize the amount of activation in each ROI, corresponding contrasts of the beta weights were extracted for all voxels in a functional ROI and then averaged, resulting in a single value for each ROI and condition for each participant. A contrast value is the activation in the condition minus activation during fixation; therefore, for each condition, the contrast value is the relative activation over the same level of fixation. The effects of text condition, and diagnostic and age groups were then analyzed for each ROI in a mixed-model repeated-measures analysis of covariance (ANCOVA) using PROC MIXED in SAS version 9.2 (SAS Institute, Cary, SC, USA) with residual maximum likelihood estimation and an unstructured covariance structure for the repeated measures. These analyses included error rates and reaction times as covariates to control for the effects of performance differences. An initial model for each ROI evaluated whether either covariate showed a significant interaction with the between-subject factors, and as this was not the case for any of the ROIs examined, ANCOVA results are reported for reduced, commonslopes models dropping these interaction terms. Results were considered statistically significant if $P<0.05$.

Functional connectivity. A measure of functional connectivity was derived for each participant in each group separately for the literal and irony text conditions. The functional connectivity was computed as a correlation between the average time course of all the activated voxels in each member of a pair of ROIs. The activation time course for each ROI was extracted separately for each participant, and was based on the normalized and smoothed images. The time courses were high-pass filtered (cutoff $128 \mathrm{sec}$ ) and had the linear trend removed. The functional connectivity values were estimated by correlating the time courses of pairs of ROIs for ten consecutive images of each trial (the first four images corresponding to a critical utterance and the remaining six images corresponding to a fixation interval between the critical utterance and the following question). This time window was used to capture the brain function related to the literal/irony comprehension while allowing for the 5-6-sec delay of the hemodynamic response. The analysis of an ROI pair eliminated any participant who had fewer than 12 activated voxels (voxel volume $8 \mathrm{~mm}^{3}$ ) in one of the ROIs. The number of excluded participants is discussed later when describing the computation of hemispheric networks. Fisher's $r$ to $z^{\prime}$ transformation was applied to the correlation coefficients for each participant prior to averaging and statistical comparison of the four 
groups. These transformed correlations were used in all reported analyses.

Because we were particularly interested in differences in the use of the relevant left hemisphere (LH) and right hemisphere $(\mathrm{RH})$ processing resources related to discourse processing, the analyses comparing functional connectivity differences between the group with autism and the typically developing controls within a developmental age group was focused on the ROI pairs, which are thought to comprise the relevant language- and text/ discourse-processing networks, based on previous research with populations with TD [Ferstl, Neumann, Bogler, \& von Cramon, 2008]. A single number was generated to index the functional connectivity of the $\mathrm{LH}$ language and RH theory-of-mind networks by averaging the z-transformed correlations between each of the ROI pairs in that network. An LH language network (ten ROI pairs) and RH theory-of-mind network (three ROI pairs) were created from the average FC of the relevant ROIs. The LH network consisted of LOPER: LSTG, LOPER: LMT, LOPER : LTRIA, LTRIA : LSTG, LTRIA : LMT, LMedFG : LTRIA, LMedFG : LOPER, LMedFG : LSTG, LMedFG : LMT, and LMT : LSTG. For these ROI pairs, one child with autism did not have a functional connectivity measure for four pairs (LOPER: LTRIA, LTRIA : LSTG, LTRIA : LMT, and LMedFG : LTRIA) in either the literal or irony text condition.

The RH theory-of-mind network consisted of LMedFG : RMT, LMedFG : RTPJ, and RMT : RTPJ. The LMedFG, RMT, and RTPJ regions have been associated with processing theory-of-mind stimuli and irony [Carrington \& Bailey, 2009; Eviatar \& Just, 2006; Frith \& Frith, 2003], suggesting that communication between these regions would be involved in our task. Connections with RPFC were not included in the $\mathrm{RH}$ network because of the large number of participants (40-50\% per group) with inadequate activation for assessing functional connectivity) for this ROI. One child with autism and one adult control had missing data for all three ROI pairs and were excluded from further analysis for this network. In addition, three children with autism and one child control were missing data for two pairs (LMedFG: RMT and RMT : RTPJ), and one adult with autism was missing data for two pairs (LMedFG : RTPJ and RMT : RTPJ) for both the literal and irony conditions.

The effects of text condition and diagnostic and age groups on functional connectivity were analyzed separately for the LH language network and the RH theoryof-mind network with mixed-model repeated-measures ANCOVAs, similar to those used for the activation data. In addition to including error rates and reaction times as covariates, these models also included activation data from each participant to control for possible effect of activation differences on functional connectivity. A measure of activation was computed by averaging the contrast values across all nodes in the LH language and the RH theory-of-mind networks, separately for each network and each condition. An initial model for each network evaluated whether any of the three covariates showed a significant interaction with the betweensubject factors. Although there were no reliable interactions involving the covariates for either network in these preliminary tests, a significant main effect of activation on functional connectivity was obtained for the $\mathrm{RH}$ network, meaning that individuals with higher activation had higher functional connectivity; however, no significant effect of activation on functional connectivity occurred in the LH network. ANCOVA results are reported for reduced, common-slopes models dropping these interaction terms. Results were considered statistically significant if $P<0.05$.

\section{Results \\ Overview}

Both the adults and children with autism had lower functional connectivity than their age- and ability-matched comparison group in the LH language network during the irony task. Unlike the child controls, neither of the autism groups had an increase in functional connectivity in response to the increasing demands of the irony condition. The children and adults with autism differed from each other in the use of key language-processing regions (LMT, LTRIA, RMT, and LMedFG), with the adults with autism having changes similar to those of the child and adult control groups.

\section{Behavioral Results}

Repeated-measures analyses of variance were used to compare the accuracy and reaction time performances in the two text conditions (literal vs. irony) by the two age groups (children vs. adults) by diagnostic group (autism vs. control). All four groups produced more errors $[\mathrm{F}(1,50)=23.53, P<0.0001]$ and had slower response times $[\mathrm{F}(1,50)=41.42, P<0.0001]$ in the irony condition as compared with the literal condition, as shown in Table 2 , suggesting that the irony text was more challenging than the literal text for all the participants. With respect to error rate, there was a main effect for diagnostic group $[\mathrm{F}(1,50)=7.33, P=0.009]$; post hoc Bonferronicorrected $t$-test analyses indicated that the adults with autism were less accurate than the control adults in the irony text condition [t $(23)=2.36, P=0.027]$. The children with autism were as accurate as the children with TD in both the literal and irony conditions.

The mean reaction times of the groups with autism were not reliably different from those of the control groups $[\mathrm{F}(1,50)=0.252, P=0.618]$. There was a main effect for age 
Table 2. Behavioral performance for the four groups on both text conditions

\begin{tabular}{|c|c|c|c|c|c|c|c|c|}
\hline \multirow[b]{2}{*}{ Children } & \multicolumn{2}{|c|}{ Error Rates } & \multirow[b]{2}{*}{$\mathrm{t}(27)$} & \multirow[b]{2}{*}{$P$} & \multicolumn{2}{|c|}{ Reaction times } & \multirow[b]{2}{*}{$\mathrm{t}(27)$} & \multirow[b]{2}{*}{$P$} \\
\hline & Autism & Control & & & Autism & Control & & \\
\hline Literal & $11.7 \% \pm 4.2$ & $7.1 \% \pm 2.7$ & 0.913 & 0.369 & $2964 \pm 145$ & $2897 \pm 147$ & 0.325 & 0.747 \\
\hline Irony & $28.0 \% \pm 3.6$ & $21.2 \% \pm 3.5$ & 1.34 & 0.192 & $3206 \pm 103$ & $3276 \pm 139$ & 0.412 & 0.684 \\
\hline Adults & & & $t(23)$ & $P$ & & & $\mathrm{t}(23)$ & $P$ \\
\hline Literal & $5.9 \% \pm 2.4$ & $3.7 \% \pm 2.1$ & 0.712 & 0.483 & $2472 \pm 114$ & $2433 \pm 130$ & 0.230 & 0.820 \\
\hline Irony & $21.2 \% \pm 5.8$ & $5.5 \% \pm 2.9$ & 2.36 & 0.027 & $2896 \pm 194$ & $2676 \pm 90$ & 1.00 & 0.328 \\
\hline
\end{tabular}

Note. Values are mean \pm standard error.

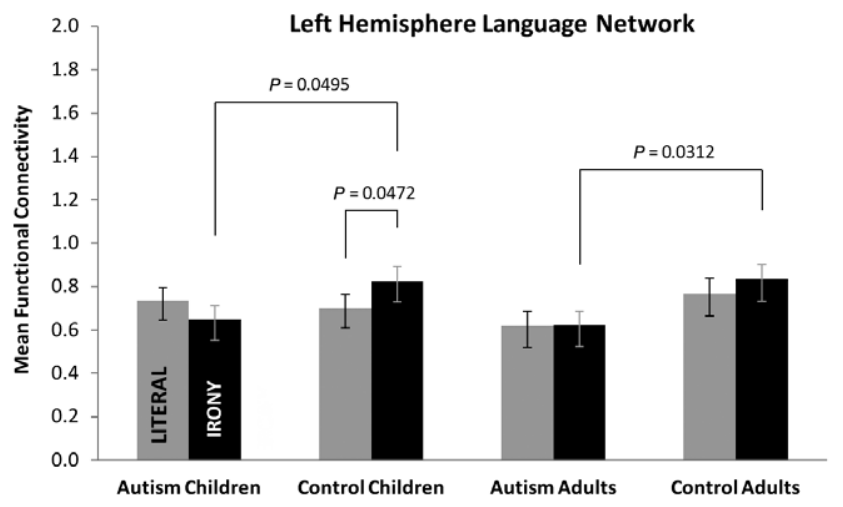

Figure 1. Mean functional connectivity for the four groups for the left hemisphere language network showing that both the children and adults with autism had lower functional connectivity than the control groups during the irony comprehension task. The child control groups also had a reliable increase in functional connectivity when comparing the literal with the irony condition. Values are least-squares means adjusted for the behavioral covariates and the activation covariate in the mixed model. Error bars represent the standard errors of these least-squares means.

group with both groups of children being reliably slower to respond than the adult groups $[\mathrm{F}(1,50)=13.33$, $P=0.001]$, but there was no interaction between diagnosis and age group $[\mathrm{F}(1,50)=0.264, P=0.610]$.

\section{Functional Connectivity}

Degree of network coordination. The differences in functional connectivity between the autism and control groups, adults and children, and across the two text conditions were analyzed separately for the LH language network and the RH theory-of-mind network. There was a main effect for diagnostic group in the LH language network $[\mathrm{F}(1,50)=4.63, P=0.0363]$, and an interaction between diagnostic group and condition $[\mathrm{F}(1,50)=5.70$, $P=0.0208$ ] (see Fig. 1). Examination of the simple effects of diagnostic group within the two text conditions revealed a reliable group difference for the irony condi- tion $[\mathrm{F}(1,50)=8.79, P=0.0046]$ but not for the literal condition. Simple effects tests within each of the age groups indicated that the adults with autism had reliably lower functional connectivity in the irony condition than the control adults $[\mathrm{F}(1,50)=4.91, P=0.0312]$, and the children with autism had reliably lower functional connectivity in the irony condition than the control children $[\mathrm{F}(1,50)=4.05, P=0.0495]$. No main effect of age group or condition was obtained nor were there any other reliable interactions. Both the children and adults with autism had lower functional connectivity than the control groups in the LH language network during the irony comprehension task. Examination of the functional connectivity for the individual pairs included in the LH language network indicated that there were no pairs in which the either the adults or children with autism had greater connectivity than the adult or child controls.

Response to text content. Although there was not a reliable three-way interaction in the LH language network, we were nevertheless interested in the withingroup differences in functional connectivity between the literal and irony condition, and therefore conducted planned contrasts of the simple effect of condition within each group. Only the control children had a reliable increase in functional connectivity between the literal and irony conditions $[\mathrm{F}(1,50)=4.14, P=0.0472]$ in the LH language network.

RH theory-of-mind network. For the RH theory-ofmind network, there were no reliable main effects for diagnostic group, age group, or text conditions, nor were there reliable interactions for diagnostic group, age group, and text condition. Levels of functional connectivity were similar across the four groups for both of the text conditions in the RH theory-of-mind network. There were no pairs included in the RH theory-of-mind network in which either the adults or children with autism had greater connectivity than the adult or child controls. 


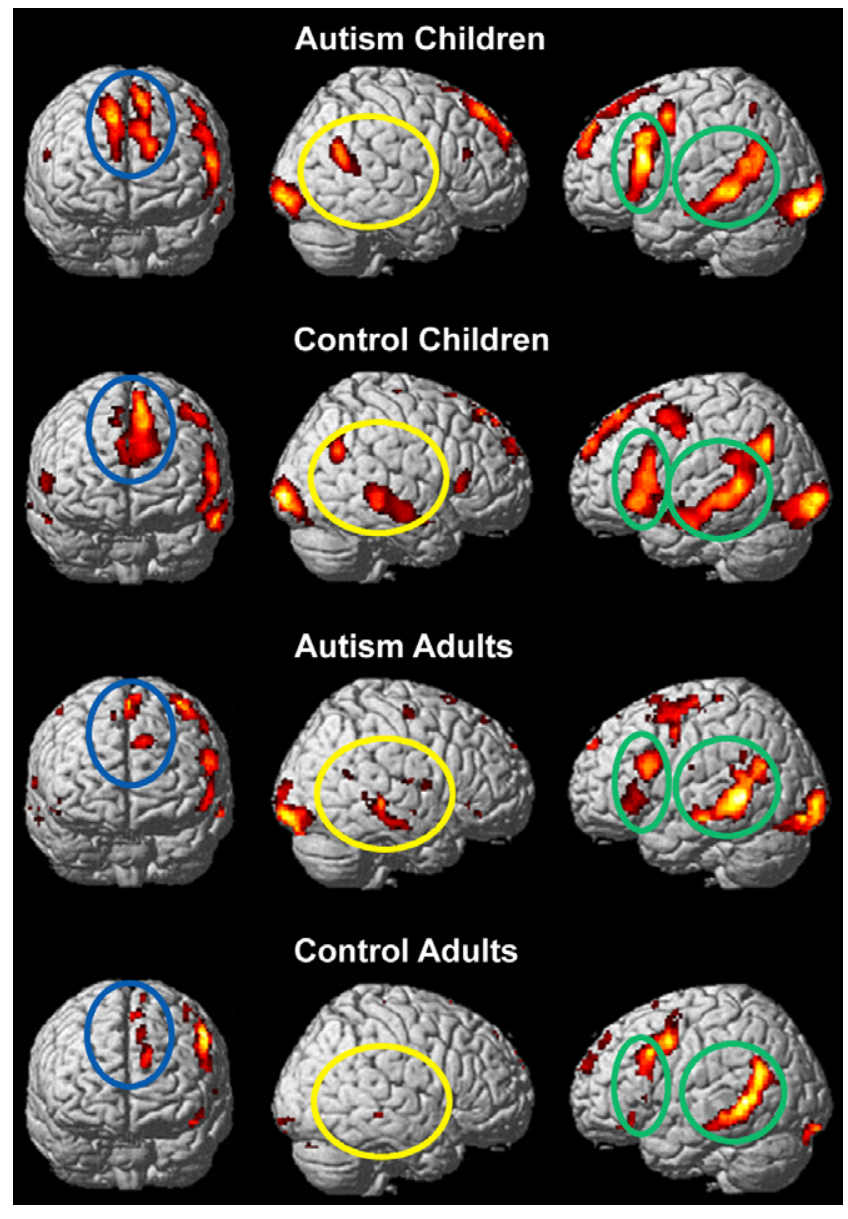

Figure 2. Within-group brain activation in the literal versus fixation condition. The children and adults with autism and the control children have activation in right hemisphere (RH) homologs not seen in the adult controls. Figures are thresholded at $P<0.001$, uncorrected. The green ellipses indicate left hemisphere language areas, the blue ellipse represents the left medial frontal region, and the yellow ellipse indicates the $\mathrm{RH}$ temporal regions.

\section{Distribution of Activation}

Within-group differences in brain activation. The t-maps for each of the groups indicating the within-group brain activation (thresholded at $P<0.001$, uncorrected) are shown in Figure 2 for the literal versus fixation condition, and in Figure 3 for the irony versus fixation condition. In both text conditions, all four groups had similar cortical activation locations; however, differences occurred in the amount of activation in a given area for each of the text conditions. The language/discourseprocessing network included activation in the expected left frontal (LOPER and LTRIA) and posterior temporal (LMT and LSTG), medial frontal (LMedFG), and right frontal (RPFC) and posterior temporal regions (RMT and RTPJ). The distribution of the workload among the network member nodes appeared to differ for both of the
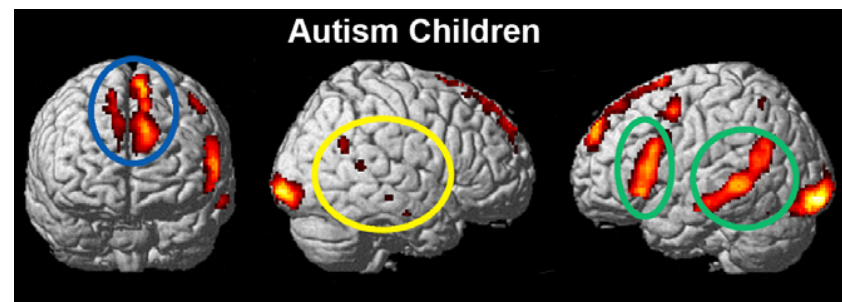

Control Children
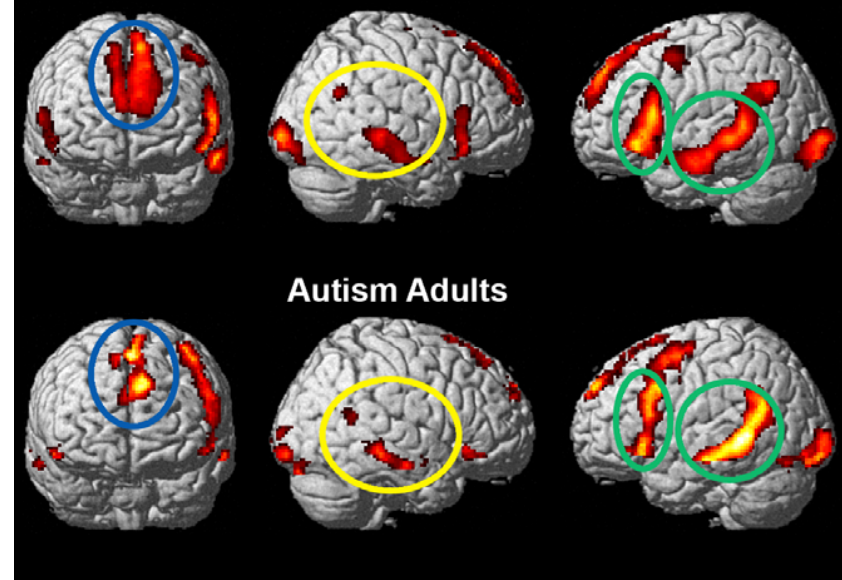

Autism Adults
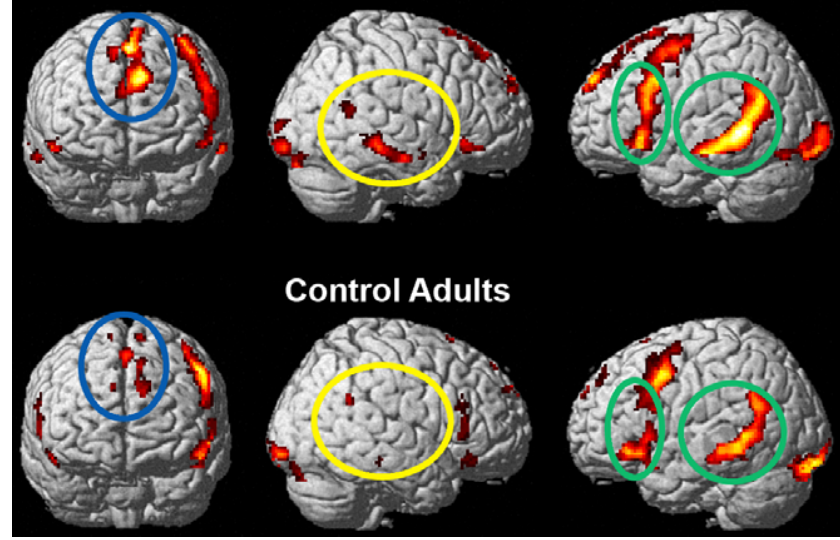

Control Adults
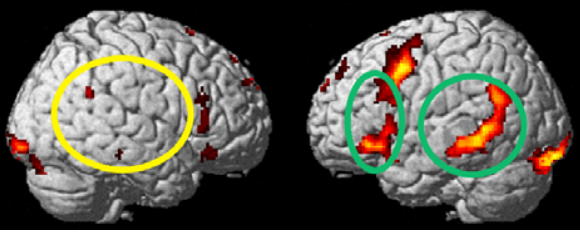

Figure 3. Within-group brain activation in the irony versus fixation condition. The language-processing areas are more left lateralized for the children with autism and the adult controls. Figures are thresholded at $P<0.001$, uncorrected. The green ellipses indicate left hemisphere language areas, the blue ellipse represents the left medial frontal region, and the yellow ellipse indicates the right hemisphere temporal regions.

autism groups as compared with their respective agematched controls for the two text conditions. Activation differences between the adults with autism and the children with autism were also evident; the children with autism had a primarily left-lateralized processing network, whereas the adults with autism, similar to the control children, have a more bilateral network for both the literal and irony conditions.

Activation Differences in Response to Text Content

Separate mixed-model ANCOVAs with error rates and reaction times as covariates were conducted for these eight ROIs relevant to discourse processing to assess the effects of diagnostic group, age, and text condition (irony vs. literal) on activation, using the mean contrast of beta weights between each text condition and fixation as the 


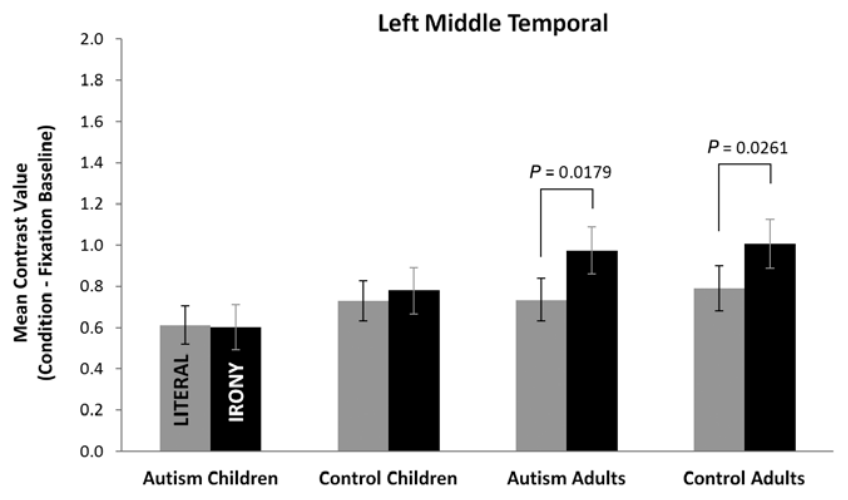

Figure 4. Mean contrast values (activation during the literal or irony conditions minus activation during fixation) for the four groups for the left middle temporal region demonstrating reliably higher activation in the adult groups than in the child groups for the irony condition. Unlike the adults, neither of the child groups had a reliable increase in activation during the irony condition. Values are least-squares means adjusted for the behavioral covariates in the mixed model. Error bars are the standard errors of these least-squares mean estimates.

dependent measure. Briefly, four of the five nodes of the left hemisphere language network showed reliably greater activation in the irony condition than in the literal condition across groups. In contrast, only the right middle temporal node of the theory-of-mind network showed this effect. Later we discuss, first, the results of these analyses for nodes of the left hemisphere language network, followed by those for the right hemisphere theory-of-mind network.

Response of LH language ROIs. The two left temporal lobe regions showed differential sensitivity to the manipulation of text condition across and within groups. No reliable main effects or interactions were obtained for the LSTG region, and additional planned contrasts of the simple effect of text condition revealed no differences in activation as a function of condition for any of the four groups. In contrast, for LMT, a main effect of condition $[\mathrm{F}(1,50)=5.22, P=0.0267]$ and an age group by text condition interaction effect $[\mathrm{F}(1,50)=5.50, P=0.0231]$ were obtained (see Fig. 4). Examination of simple effects indicated that there was an age group effect within the irony text condition $[\mathrm{F}(1,50)=6.53, P=0.0137]$; the adult groups had more activation in LMT than the child groups during the irony condition. Planned contrasts examining the simple effect of text condition within each of the four groups revealed that both the adults with autism $[\mathrm{F}(1,50)=6.00, \quad P=0.0179]$ and the control adults $[\mathrm{F}(1,50)=5.25, P=0.0261]$ had reliably greater activation in the irony than the literal condition, but this was not true for either group of children.

Both of the frontal lobe nodes of the LH language network (LTRIA and LOPER) showed effects of the text

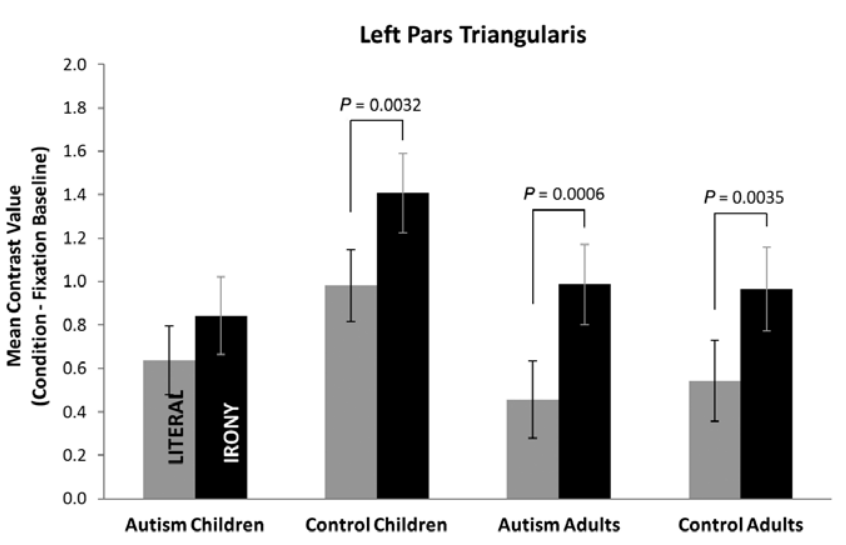

Figure 5. Mean contrast values for the four groups for left pars triangularis showing reliably increased activation for both of the control groups and the adults with autism for the irony condition, but not for the children with autism. Values are least-squares means adjusted for the behavioral covariates in the mixed model. Error bars show the standard error of these least-squares mean estimates.

condition manipulation. A strong main effect of condition was obtained for LTRIA $[\mathrm{F}(1,50)=24.14, P<0.0001]$, although this did not interact with diagnosis or age, nor were there reliable main effects of these factors (see Fig. 5). Nevertheless, planned contrasts of the simple effect of text condition within each group revealed that the adults with autism $[\mathrm{F}(1,50)=13.49, P=0.0006]$, the control adults $[\mathrm{F}(1,50)=9.42, P=0.0035]$, and the control children $[\mathrm{F}(1,50)=9.63, P=0.0032]$ had reliably greater activation in the irony than the literal condition for this region; but no reliable difference between the two conditions was obtained for the children with autism. For LOPER, a main effect of condition $[\mathrm{F}(1,50)=4.59, P=0.0371]$ was obtained, but no other reliable differences occurred.

Response of the RH theory-of-mind ROIs. Among the right hemisphere nodes of the theory-of-mind network, only the RMT region was responsive to the effect of text condition with a main effect of condition $[\mathrm{F}(1,50)=4.81, P=0.0329]$ obtained for this region (see Fig. 6). Examination of the simple effect of condition within each group revealed that only the control adults had reliably greater activation in RMT for the irony condition relative to the literal condition $[\mathrm{F}(1,50)=6.81$, $P=0.0119]$. In addition, there was a reliable simple effect of age within the autism group for the irony condition $[\mathrm{F}(1,50)=6.96, P=0.0111]$, indicating that the adults with autism had reliably greater activation in this region than the children with autism. None of the four groups had reliably different activation for RTPJ for either the literal or irony text conditions. No reliable differences were obtained for RPFC; all four groups had relatively low levels of activation in this region for both text conditions. 


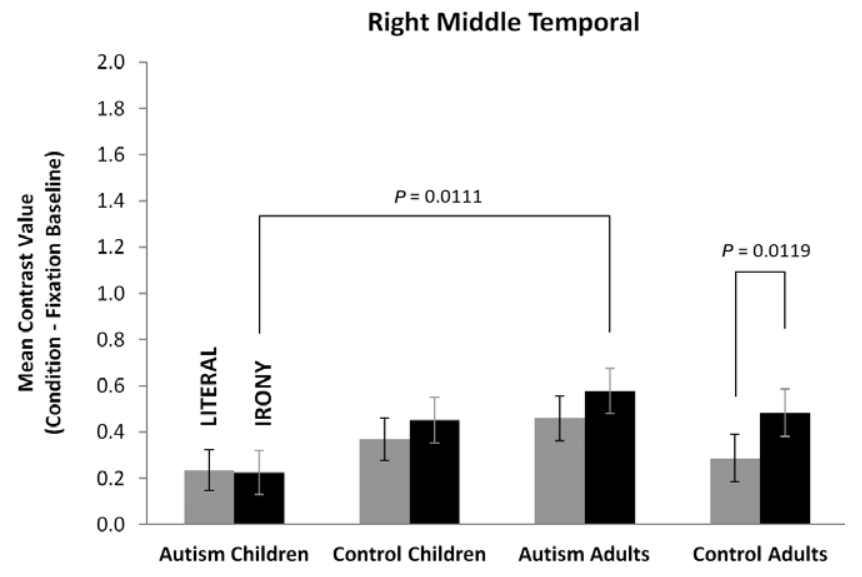

Figure 6. Mean contrast values for the four groups for the right middle temporal region showing that the children with autism have reliably lower activation than the adults with autism, and that the adult controls have a reliable increase in this region for irony processing. Values are least-squares means adjusted for the behavioral covariates in the mixed model. Error bars are standard errors of these least-squares means.

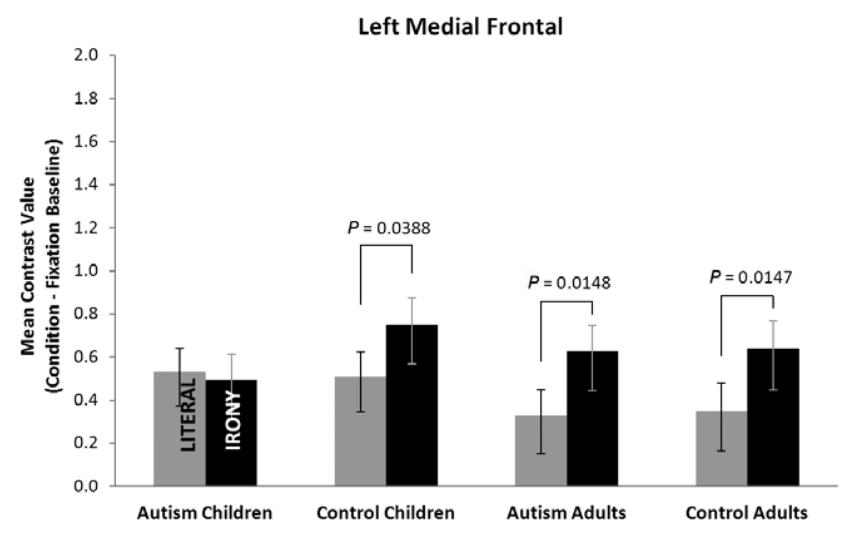

Figure 7. Mean contrast values for the four groups for left medial frontal gyrus showing reliably increased activation for both the children and adults controls, and the adults with autism for the irony condition, but not for the children with autism. Values are leastsquares means adjusted for the behavioral covariates in the mixed model. Error bars are standard errors of the least-squares means.

Left medial frontal region response. The left medial frontal ROI participates in both networks and may serve an integrative role in discourse processing. A main effect of text condition $[\mathrm{F}(1,50)=9.2, P=0.0038]$ occurred for LMedFG, but did not interact with diagnosis or age, and there were no reliable main effects of these factors (see Fig. 7). However, examination of planned contrasts of the simple effect of text condition within each group indicated that the adults with autism $[\mathrm{F}(1,50)=6.38$, $P=0.0148]$, the control adults $[\mathrm{F}(1,50)=6.38, \quad P=$ 0.0147], and the control children $[\mathrm{F}(1,50)=4.5, P=$
0.0388] had reliably greater activation in the irony than the literal condition for this region.

\section{Discussion}

Comparison of neural function in the same language tasks, with a variation in text content, with both children and adults with autism and child and adult controls can reveal aspects of language processing that are characteristic of the disorder while providing insight into differences related to developmental status. Overall, both the children and adults with autism had lower coordination within the left hemisphere language network during irony comprehension (the most demanding language task) as compared with the age- and ability-matched controls. In addition, unlike the children with TD, neither the children nor the adults with autism had an increase in functional connectivity in the irony text relative to the literal text, even though their behavioral performance indicated that all three groups were challenged by this task. Examination of activation in brain regions related to language and theory of mind processing revealed that, in general, the children and adults with autism used a language/discourse-processing network that incorporated the same left and right hemisphere cortical regions previously reported for individuals with TD [Ferstl et al., 2008], including LOPER, LTRIA, LSTG, LMT and RMT, LMedFG, RTPJ, and RPFC. However, the distribution of the workload among the member nodes differed for the adults with autism (as compared with the adult controls) and for children with autism (as compared with the child controls) for both the literal and irony text conditions. Finally, age-group differences occurred for the autism groups with respect to the dynamic recruitment of cortical regions in response to text content, particularly LMT and RMT for the adults with autism, and LTRIA and LMedFG for the children with autism. These findings indicate variations related to developmental level within the diagnostic group.

The relatively lower functional connectivity of the children and adults with autism in the left hemisphere language network during irony comprehension is consistent with previous studies reporting underconnectivity for adults with autism in language-processing tasks [Just et al., 2004; Kana et al., 2006; Mason et al., 2008] and with other studies reporting reduced functional connectivity in cognitive tasks that require coordination between frontal-posterior cortical areas [see review in Schipul, Keller, \& Just, 2011]. Furthermore, both the children and adults with autism failed to show an increase in functional connectivity in response to the irony text relative to the literal text. Functional connectivity is thought to be an index of the adaptability of a cortical network or the ability to modify synchronization in the face of 
changing task demands [Prat, Keller, \& Just, 2007]. In individuals with TD, more demanding conditions generally produce higher functional connectivity than similar less demanding conditions [Diwadkar, Carpenter, \& Just, 2000; Hampson, Peterson, Skudlarski, Gatenby, \& Gore, 2002], the pattern displayed by the child controls in the left hemisphere language network when comparing the irony condition to the literal condition. However, neither of the autism groups had a statistically reliable increase in functional connectivity from the literal to the irony condition in the left hemisphere language network, even though the behavioral measures indicated that the irony task was relatively challenging for all three of these groups. The lack of an increase in functional connectivity on the part of the autism participants suggests less adaptability or responsiveness to the differing task demands.

The lack of an increase in functional connectivity or cortical synchronization for the irony condition by both the adults and children with autism could occur for several reasons. One interpretation is that there is a biological constraint that limits the flow of communication between the key cortical regions secondary to cortical abnormalities that interfere with interhemisphere and intrahemispheric communication. A number of such abnormalities such as increased density of neuronal cells with smaller and more numerous minicolumns [Casanova, Buxhoeveden, Switala, \& Roy, 2002; Casanova et al., 2006] and enlargement of white matter [Herbert et al., 2004] have been reported in the brains of individuals with autism. In addition, studies using diffusion tensor imaging (DTI) have reported lower fractional anisotropy (a measure of the coherence of diffusion directionality) in adolescents with autism in frontal-temporal pathways, suggesting decreased white matter integrity [Sahyoun, Belliveau, Soulières, Schwartz, \& Mody, 2010]. Other DTI studies have found results consistent with decreased white matter integrity in both adolescents and children with ASD in the arcuate fasciculus, which connects frontal and posterior language regions [Fletcher et al., 2010; Kumar et al., 2010]. Reductions in the structural integrity of white matter in autism have also been reported to persist into adulthood [Keller, Kana, \& Just, 2007], with decreased white matter volume reported for frontal connections, including the uncinate fasciculus and fronto-occipital fasciculus, and the arcuate fasciculus connecting the Broca and Wernicke areas [Ecker et al., 2012]. Therefore, the functional underconnectivity in the children and adults with autism could be related to underlying problems with the structural integrity of white matter connections between frontal and posterior brain regions.

A second less likely interpretation for the lack of a relative increase in functional connectivity in the autism groups is that the children and adults failed to distinguish between the irony passages and the literal ones, using the same strategies and resources in processing both types of passages. One argument against this latter interpretation, at least for the adults with autism, is that an activation increase did occur during irony processing in relevant areas (LMT and RMT, and LMedFG), indicating that the two types of passages were being processed differently. Furthermore, behavioral studies suggest that the overall pattern of response by individuals on the autism spectrum to different types of nonliteral language is the same as that of individuals with TD, for example, erring more on novel than on familiar metaphors [Giora, Gazal, Goldstein, Fein, \& Stringaris, 2012].

Although not the main focus of this study, consideration of the differences between the control adults and the control children provides a context for the understanding of the differences between the adults and children with autism. The control adults had a primarily left-lateralized language-processing network for both the irony and the literal conditions. However, the language network for the control children was bilateral for both language conditions. In addition, the control adults had a very specific response, reliably increased activation in the left middle temporal language-processing region and the right middle temporal region, for the irony context as compared with the literal context that was not observed in the control children. Like the control adults, the control children had reliably increased activation in LTRIA and left medial frontal regions in comparing the literal with the irony contexts. The control children had a reliable increase in functional connectivity in comparing the literal with the irony condition that, although expected, was not observed in the control adults. In general, the control adults had a less distributed, more left-lateralized language-processing network than the control children, with focused increases in activation in response to the increasing demands of the languageprocessing tasks but did not have the expected increase in functional connectivity when processing ironic content.

Like the adult controls, the adults with autism had increased activity in the LMT in the irony condition relative to the literal condition. The increased activity in the LMT may be due to a general age effect, as this result was obtained for both adult groups but did not occur for either child group. This finding is consistent with prior reports that this region is sensitive to maturational differences with greater activation occurring with increasing age [Chou et al., 2006]. An increase in skill and learning on semantic tasks, and an increase in elaboration of semantic representations have also been associated with greater activation in the LMT [Blumenfeld, Booth, \& Burman, 2006; Sandak, Mencl, \& Frost, 2004], further indications that this is an area that is sensitive to linguistic experience.

An age-related difference that was specific to the autism group was obtained for the level of activation in the RMT 
during the irony condition; the adults with autism had relatively greater activation than the children with autism in this region when reading the irony texts. The control adults did not have reliably greater activation in this region than the control children; however, they did have a reliable increase in activation in the RMT when comparing the literal with irony conditions. Prior research suggests that the right temporal region is related to context processing [Vigneau et al., 2011], a relevant cognitive process for the interpretation of irony. The relative increase in activation from the literal to the irony condition for the adult controls also supports its significance to the processing of ironic text. Unlike the children with autism, the adults with autism may have used contextual knowledge, gleaned from their relatively greater level of experience, for the construction of meaning during the irony condition. However, unlike the adult controls, the adults with autism did not have a reliable increase in RMT activation in this region in response to the demands of the irony task. This lack of selective activation of a relevant brain region is similar to previously reported results of another fMRI study with adults with autism in which they had a lack of differential use of right hemisphere regions during discourse-processing tasks [Mason et al., 2008].

Both of the adults groups and the child controls had a reliable increase in activation in the LTRIA and LMedFG, whereas the children with autism did not. This pattern suggested some normalization of function in these regions with increasing age and linguistic experience for the adults with autism. Similar to the current results, Colich et al. [2012] reported relatively less activation in both LTRIA and LOPER for a group of adolescents with ASD (mean age 14.27 years) as compared with an agematched TD group (mean age 13.15 years) when viewing visual scenes and making judgments about auditorily presented ironic statements. Groen et al. [2010] also noted a similar lack of activation in LTRIA by individuals with autism, 12-18 years of age, in a task that required the integration of social information. The LTRIA is a region that has been associated with semantic processing [Friederici, Opitz, \& von Cramon, 2000], including a greater search for semantic associations [Chou et al., 2006] or the selection between competing representations [Hirshorn \& Thompson-Schill, 2006]. The lack of an increase in activation in this region for irony processing on the part of the children with autism may indicate a reduced lack of appreciation of the ironic information. That is, they may have failed to realize that a competing meaning was possible and, therefore, did not always search for an alternative meaning.

Alternately, the difference in level of activation for the children with autism in the LTRIA may be reflective of underlying anatomical differences that are constraining the level of available resources. At least one study [Knaus et al., 2009] has reported an increase in volume in LTRIA and LOPER in children with autism at ages similar to those in the current study. In this scenario, the increase in volume is not equated with an increase in neurofunction, but rather an abnormality in neural development that in turn negatively impacts neural processing.

With the right temporal regions, the medial frontal gyrus is thought to play a key role in discourse comprehension and theory-of-mind tasks [see review Mar, 2011] and is also thought to be part of a general inference network [Mason \& Just, 2011]. The results from the current study would support a central role for the left medial frontal cortex in irony processing, as greater recruitment of this area occurred during the irony condition for both adult groups and the child controls. The medial frontal gyrus is a region in which activation levels for children with ASD have been previously reported to be both similar [Wang et al., 2006] and greater [Colich et al., 2012] than children and adolescents with TD when processing auditorily presented ironic remarks. However, the tasks in both of those studies explicitly asked the children to interpret a character's communicative intentions and required additional attention to auditorily presented information. Therefore, the children in those studies may have directed more effort toward that process, resulting in the recruitment of this brain region. The lack of differential activation of the LMedFG by the children with autism in the current study may reflect reduced theory-of-mind or inferential abilities, or it may reflect the lack of explicit cues that would trigger the use of these cognitive resources. The activation pattern of the adults with autism for this region was similar to that of the child and adult controls, suggesting increased normalization of this region with age and experience.

\section{Conclusions}

Both the adults and children with autism differed from the adult and child controls in (a) the degree of network coordination, (b) the distribution of the workload among the parts of the network, and (c) in the active recruitment of key brain regions for the processing of the ironic texts. Differences in cortical activation between the two autism age groups suggested positive effects in language functioning with age. However, as indexed by the functional connectivity measures, regardless of age, the participants with autism had less adaptability or responsiveness to the differing task demands than the child and adult controls. The behavioral cost of the inefficiency of processing indexed by the functional connectivity measure was seen only when comparing the performance of the two adult groups, when the adults with autism performed significantly worse than the adult controls. The importance of network coordination to linguistic processing was sup- 
ported by the relatively minimal activation differences between the two adult groups; it was the coordination between the nodes of the network that was significantly different, not the activity in individual brain regions. The adults with autism may have had increased semantic knowledge (as indicated by LMT activation) relative to both child groups, increased contextual knowledge (as indicated by RMT activation), and increased coherence processing (as indicated by LMedFG activation) relative to the children with autism, but these functional differences did not result in relatively better behavioral performance than either of the child groups. Whereas activation levels appeared to somewhat normalize with age for text comprehension, the problems with network coordination were persistent. Concurrent with the network coordination problems of the adults with autism was a relatively poorer performance on the behavioral measures of irony comprehension.

Because this is a cross-sectional rather than a longitudinal study, the conclusions that can be drawn about the developmental process in autism are limited. However, differences in the neural response between the children and adults with autism provides evidence that positive changes may occur in brain function with maturation and experience in this neurodevelopmental disorder. Improvements in cognitive skills from childhood to adulthood in autism have been previously reported in a cross-sectional study of executive function [Luna, Doll, Hegedus, Minshew, \& Sweeney, 2007]; the results of our study suggest similar improvements associated with linguistic development. These positive differences suggest that, in autism just as in TD, neurofunctional changes do occur as part of the developmental process in response to environmental input. However, the results of the current study also indicate that adults with autism have continuing challenges with more demanding linguistic processing tasks as indicated by the persistent underconnectivity in the left hemisphere language network.

\section{Clinical Implications}

The results of this study suggest that, during text comprehension, verbal, high-functioning children and adults with autism have challenges in the coordination of cortical regions in the left hemisphere language network. This reduced efficiency in processing may make them susceptible to overloads when the demand for cognitive processing increases. In addition, both children and adults with autism may not realize when a different cognitive strategy needs to be used for textual comprehension. External measures such as management of the amount of information presented and the use of explicit cues as to the type of textual structure may help individuals with autism manage these demands. For example, Mashal and Kasirer [2011] reported positive results when using a visual "thinking maps" strategy to teach novel metaphors to children with autism. However, even with these types of measures, underlying neurofunctional differences may limit the ability of the children to process the textual information, meaning they must rely upon semantic and contextual knowledge to perform the discourse-processing task to a greater extent than expected given their overall level of cognitive functioning. The assumption of reliance on semantic knowledge for irony processing is consistent with the results of Mashal and Kasirer [2012] that suggested that semantic knowledge and reading fluency were fundamental skills for the comprehension of metaphors in children with ASD.

\section{Acknowledgments}

The authors are grateful to the participants and their families for their commitment to this research. Thanks are also extended to AnnaMaria Tomlanovich for assistance with data analysis, to Sarah Schipul for assistance with data collection, to Kara Cohen for preparation of the figures, and to Chantel Prat for providing useful comments on an earlier version of the manuscript. The authors have no conflict of interests to declare.

\section{References}

Baron-Cohen, S., Leslie, A.M., \& Frith, U. (1985). Does the autistic child have a "theory of mind"? Cognition, 21, 37-46.

Blumenfeld, H.K., Booth, J.R., \& Burman, D.D. (2006). Differential prefrontal-temporal neural correlates of semantic processing in children. Brain and Language, 99, 226-235.

Booth, J.R., Burman, D.D., Meyer, J.R., Gitelman, D.R., Parrish, T.B., \& Mesulam, M.M. (2004). Development of brain mechanisms for processing orthographic and phonologic representations. Journal of Cognitive Neuroscience, 16, 1234-1249.

Carrington, S.J., \& Bailey, A.J. (2009). Are there theory of mind regions in the brain? A review of the neuroimaging literature. Human Brain Mapping, 30, 2313-2335.

Casanova, M.F., Buxhoeveden, D., Switala, A., \& Roy, E. (2002). Minicolumnar pathology in autism. Neurology, 58, 428-432.

Casanova, M.F., van Kooten, I.A.J., Switala, A.E., van Engeland, H., Heinsen, H., et al. (2006). Minicolumnar abnormalities in autism. Acta Neuropathologica, 112, 287-303.

Chou, T.-L., Booth, J.R., Burman, D.D., Bitan, T., Bigio, J.D., et al. (2006). Developmental changes in the neural correlates of semantic processing. NeuroImage, 29, 1141-1149.

Colich, N.L., Wang, A.T., Rudie, J.D., Hernandez, L.M., Bookheimer, S.Y., \& Dapretto, M. (2012). Atypical neural processing of sarcastic and sincere remarks in children and adolescents with autism spectrum disorders. Metaphor and Symbol, 27, 70-92.

Diwadkar, V.A., Carpenter, P.A., \& Just, M.A. (2000). Collaborative activity between parietal and dorso-lateral prefrontal cortex in dynamic spatial working memory revealed by fMRI. NeuroImage, 12, 85-99. 
Ecker, C., Suckling, J., Deoni, S.C., Lombardo, M.V., Bullmore, E.T., et al. (2012). Brain anatomy and its relationship to behavior in adults with autism spectrum disorder: A multicenter magnetic resonance imaging study. Archives of General Psychiatry, 69, 195-209.

Eviatar, Z., \& Just, M.A. (2006). Brain correlates of discourse processing: An fMRI investigation of irony and conventional metaphor comprehension. Neuropsychologia, 44, 23482359.

Ferstl, E.C., Neumann, J., Bogler, C., \& von Cramon, D.Y. (2008). The extended language network: A meta-analysis of neuroimaging studies on text comprehension. Human Brain Mapping, 29, 581-593.

Fletcher, P.T., Whitaker, R.T., Tao, R., DuBray, M.B., Froehlich, A., et al. (2010). Microstructural connectivity of the arcuate fasciculus in adolescents with high-functioning autism. NeuroImage, 51, 1117-1125.

Friederici, A.D., Opitz, B., \& von Cramon, D.Y. (2000). Segregating semantic and syntactic aspects of processing in the human brain: An fMRI investigation of different word types. Cerebral Cortex, 10, 698-705.

Friston, K., Ashburner, J., Frith, C., Poline, J.-B., Heather, J., \& Frackowiak, R. (1995). Spatial registration and normalization of images. Human Brain Mapping, 2, 165-189.

Frith, U., \& Frith, C.D. (2003). Development and neurophysiology of mentalizing. Philosophical Transactions of the Royal Society B: Biological Sciences, 358, 459-473.

Giora, R., Gazal, O., Goldstein, I., Fein, O., \& Stringaris, K.A. (2012). Salience and context: Interpretation of metaphorical and literal language by young adults diagnosed with Asperger's syndrome. Metaphor and Symbol, 27, 22-54.

Groen, W.B., Tesink, C., Petersson, K.M., van Berkum, J., van der Gaag, R.J., et al. (2010). Semantic, factual, and social language comprehension in adolescents with autism: An FMRI study. Cerebral Cortex, 20, 1937-1945.

Hampson, M., Peterson, B.S., Skudlarski, P., Gatenby, J.C., \& Gore, J.C. (2002). Detection of functional connectivity using temporal correlations in MR images. Human Brain Mapping, $15,247-262$.

Harris, G.J., Chabris, C.F., Clark, J., Urban, T., Aharon, I., et al. (2006). Brain activation during semantic processing in autism spectrum disorders via functional magnetic resonance imaging. Brain and Cognition, 61, 54-68.

Herbert, M.R., Ziegler, D.A., Makris, N., Filipek, P.A., Kemper, T.L., et al. (2004). Localization of white matter volume increase in autism and developmental language disorder. Annals of Neurology, 55, 530-540.

Hirshorn, E.A., \& Thompson-Schill, S.L. (2006). Role of the left inferior frontal gyrus in covert word retrieval: Neural correlates of switching during verbal fluency. Neuropsychologia, 44, 2547-2557.

Just, M.A., Cherkassky, V.L., Keller, T.A., \& Minshew, N.J. (2004). Cortical activation and synchronization during sentence comprehension in high-functioning autism: Evidence of underconnectivity. Brain, 127, 1811-1821.

Kana, R.K., Keller, T.A., Cherkassky, V.L., Minshew, N.J., \& Just, M.A. (2006). Sentence comprehension in autism: Thinking in pictures with decreased functional connectivity. Brain, 129, 2484-2493.
Kana, R.K., Keller, T.A., Cherkassky, V.L., Minshew, N.J., \& Just, M.A. (2009). Atypical frontal-posterior synchronization of Theory of Mind regions in autism during mental state attribution. Social Neuroscience, 4, 135-152.

Kana, R.K., Keller, T.A., Minshew, N.J., \& Just, M.A. (2007). Inhibitory control in high-functioning autism: Decreased activation and underconnectivity in inhibition networks. Biological Psychiatry, 62, 198-206.

Keller, T.A., Kana, R.K., \& Just, M.A. (2007). A developmental study of the structural integrity of white matter in autism. NeuroReport, 18, 23-27.

Knaus, T.A., Silver, A.M., Dominick, K.C., Schuring, M.D., Shaffer, N., et al. (2009). Age-related changes in the anatomy of language regions in autism spectrum disorders. Brain Imaging and Behavior, 3, 51-63.

Knaus, T.A., Silver, A.M., Lindgren, K.A., Hadjikhani, N., \& Tager-Flusberg, H. (2008). FMRI activation during a language task in adolescents with ASD. Journal of the International Neuropsychological Society, 14, 967979.

Kumar, A., Sundaram, S.K., Sivaswamy, L., Behen, M.E., Makki, M.I., et al. (2010). Alterations in frontal lobe tracts and corpus callosum in young children with autism spectrum disorder. Cerebral Cortex, 20, 2103-2113.

Lord, C., Risi, S., Lambrecht, L., Cook, E.H.J., Leventhal, B.L., et al. (2000). The Autism Diagnostic Observation ScheduleGeneric: A standard measure of social and communication deficits associated with the spectrum of autism. Journal of Autism and Developmental Disorders, 30, 205-223.

Lord, C., Rutter, M., \& LeCouteur, A. (1994). Autism Diagnostic Interview-Revised: A revised version of a diagnostic interview for caregivers of individuals with possible pervasive developmental disorders. Journal of Autism and Developmental Disorders, 24, 659-685.

Luna, B., Doll, S.K., Hegedus, S.J., Minshew, N.J., \& Sweeney, J.A. (2007). Maturation of executive function in autism. Biological Psychiatry, 61, 474-481.

Mar, R.A. (2011). The neural bases of social cognition and story comprehension. Annual Review of Psychology, 62, 103134.

Mashal, N., \& Kasirer, A. (2011). Thinking maps enhance metaphoric competence in children with autism and learning disabilities. Research in Developmental Disabilities, 32, 20452054.

Mashal, N., \& Kasirer, A. (2012). Principal component analysis study of visual and verbal metaphoric comprehension in children with autism and learning disabilities. Research in Developmental Disabilities, 33, 274-282.

Mason, R.A., \& Just, M.A. (2011). Differentiable cortical networks for inferences concerning people's intentions versus physical causality. Human Brain Mapping, 32, 313-329.

Mason, R.A., Williams, D.L., Kana, R.K., Minshew, N., \& Just, M.A. (2008). Theory of mind disruption and recruitment of the right hemisphere during narrative comprehension in autism. Neuropsychologia, 46, 269-280.

Power, J.D., Barnes, K.A., Snyder, A.Z., Schlaggar, B.L., \& Petersen, S.E. (2012). Spurious but systematic correlations in functional connectivity MRI networks arise from subject motion. NeuroImage, 59, 2142-2154. 
Prat, C.S., Keller, T.A., \& Just, M.A. (2007). Individual differences in sentence comprehension: A functional magnetic resonance imaging investigation of syntactic and lexical processing demands. Journal of Cognitive Neuroscience, 19, 19501963.

Reitan, R.M. (1985). Halstead-reitan neuropsychological test battery. Tucson, AZ: Reitan Neuropsychological Laboratories, University of Arizona.

Sachs, B.C., \& Gaillard, W.D. (2003). Organization of language networks in children: Functional magnetic resonance imaging studies. Current Neurology and Neuroscience Reports, 3, 157-162.

Sahyoun, C.P., Belliveau, J.W., Soulières, I., Schwartz, S., \& Mody, M. (2010). Neuroimaging of the functional and structural networks underlying visuospatial vs. linguistic reasoning in high-functioning autism. Neuropsychologia, 48, 86-95.

Sandak, R., Mencl, W.E., \& Frost, S.J. (2004). The neurobiology of adaptive learning in reading: A contrast of different training conditions. Cognitive, Affective, \& Behavioral Neuroscience, 4, 67-88.

Schipul, S.E., Keller, T.A., \& Just, M.A. (2011). Inter-regional brain communication and its disturbance in autism. Frontiers in System Neuroscience, 5, 1-11.
Sperber, D., \& Wilson, D. (1981). Irony and the use-mention distinction. In P. Cole (Ed.), Radical pragmatics (pp. 295-318). New York: Academic Press.

Sperber, D., \& Wilson, D. (1995). Relevance: Communication and cognition, 2nd ed. Oxford: Blackwell.

Tesink, C.M.J.Y., Buitelaar, J.K., Petersson, K.M., van der Gaag, R.J., Kan, C.C., et al. (2009). Neural correlates of pragmatic language comprehension in autism spectrum disorders. Brain, 132, 1941-1942.

Tzourio-Mazoyer, N., Landeau, B., Papathanassiou, D., Crivello, F., Etard, O., et al. (2002). Automated anatomical labeling of activations in SPM using a macroscopic anatomical parcellation of the MNI MRI single subject brain. NeuroImage, 15, 273-289.

Vigneau, M., Beaucousin, V., Hervé, P.-Y., Jobard, G., Petit, L., et al. (2011). What is right-hemisphere contribution to phonological, lexico-semantic, and sentence processing? Insights from a meta-analysis. NeuroImage, 54, 577-593.

Wang, A.T., Lee, S.S., Sigman, M., \& Dapretto, M. (2006). Neural basis of irony comprehension in children with autism: The role of prosody and context. Brain, 129, 932-943.

Wechsler, D. (1999). Wechsler abbreviated scale of intelligence. San Antonio, TX: Psychological Corporation. 